\title{
Electronic malnutrition risk screening: new directions in clinical practice
}

\author{
C. Green, L. Pearce, G. K. Hart, H. Davis and S. Ioannidis \\ Austin Health, 145 Studley Road, Heidelberg, Melbourne 3084, Australia
}

\begin{abstract}
Malnutrition is well documented in hospital patients and is associated with increased infection rates, pressure ulcers, poor wound healing and increased length of stay. Early detection (via routine screening) and subsequent treatment has been shown to improve clinical outcomes $^{(1)}$. In the modern health care environment, utilisation of the electronic health record offers the clinician significant benefits including increased time efficiencies, streamlined data collection and timely access to audit and outcome data.
\end{abstract}

This study aimed to better identify malnutrition risk at an acute tertiary hospital by:

- Developing an electronic system for malnutrition risk screening and referral.

- Demonstrating the benefits of an electronic system on routine clinical processes.

- Assessing the impact of implementing an electronic system on malnutrition risk identification and subsequent Dietitian referral rates for high risk patients.

An electronic version of a malnutrition screening tool was developed for use by nursing staff during routine admission procedure. The tool was based on the Malnutrition Universal Screening Tool ${ }^{(2)}$. The 'Malnutrition Universal Screening Tool' ('MUST') was reproduced here with the kind permission of BAPEN (British Association for Parenteral and Enteral Nutrition). The benefits of the Electronic Malnutrition Universal Screening Tool (eMUST) include: user-friendly data entry screens, routine calculation of risk score and, most importantly, an automatic link to the internal paging system.

Random samples of patients admitted to a general medical ward were screened for malnutrition risk. Patients identified at high risk were then compared against dietetic referrals received prior to $(n=96)$, and 12 months post $(n=100)$, implementation of routine electronic malnutrition risk screening.

Prior to implementation, $26 \%$ of patients screened were classified as being at high risk of malnutrition. This was consistent postimplementation with $24 \%$ of patients detected at high risk. Prior to implementation, only $24 \%$ of high risk patients had been referred to a dietitian by nursing staff. Post-implementation, $58 \%$ of high risk patients were referred to the dietitian. $(P=0.029)$.

The eMUST has been successfully incorporated into routine practice in a general medical ward at an acute tertiary hospital, which has resulted in the improved identification of high malnutrition risk patients and subsequent dietetic referral.

The successful implementation of the eMUST has led to our future intention to continue to utilise this technology within other clinical areas as well as to demonstrate the portability of this concept to other risk screening domains (i.e. pressure ulcers, falls, aggression, etc.).

1. Sorensen J, Kondrup J, Prokopowicz J et al. (2008) Clin Nutr 27, 340-349.

2. Todorovic V, Russell C, Stratton R et al. (2003) The 'MUST' Explanatory Booklet: A Guide to the 'Malnutrition Universal Screening Tool' (MUST) for Adults. Redditch, UK: The Malnutrition Advisory Group: A Standing Committee of British Association of Parenteral and Enteral Nutrition. http:// www.bapen.org.uk 